www.jmscr.igmpublication.org

Impact Factor 5.84

Index Copernicus Value: 71.58

ISSN (e)-2347-176x ISSN (p) 2455-0450

crossref DOI: _https://dx.doi.org/10.18535/jmscr/v5i10.11

Journal Of Medical Science And Clinical Research

IGM Publication

An official Publication of IGM Publication

\title{
Inter and Intra Observer Reliability of RUST score in Open Tibial Fractures treated with Intramedullary Nailing
}

\author{
Authors \\ Neeti Aggarwal $^{1}$, Vineet Aggarwal ${ }^{2}$, Rudra Pratap Singh Thakur ${ }^{3}$, Sanjiv Sharma ${ }^{4}$ \\ ${ }^{1}$ Associate Professor, Department of Radiodiagnosis Indira Gandhi Medical College, Shimla, India \\ ${ }^{2}$ Professor, Department of Orthopedics, Indira Gandhi Medical College, Shimla, India \\ ${ }^{3}$ Postgraduate student,Department of Orthopedics, Indira Gandhi Medical college, Shimla \\ ${ }^{4}$ Professor and Head, Department of Radiodiagnosis, Indira Gandhi Medical College, Shimla \\ Corresponding Author \\ Vineet Aggarwal
}

Professor, Department of Orthopedics, Indira Gandhi Medical College, Shimla, India

\begin{abstract}
Introduction: The Radiological healing score for tibia is a useful instrument to quantify fracture healing in tibial fractures. It is a validated score showing strong to almost perfect agreement between observers. The present study tends to evaluate the results of the score when applied on open tibial fractures stabilized with an intramedullary device.

Material and Methods: 60 cases of open tibial fractures that underwent intramedullary nailing were evaluated for fracture healing using the RUST score. 40 prospective cases and 20 retrospective cases were evaluated radiographically using standardized radiographs. Four observers, two of whom were orthopedic surgeons and two were radiologists, independently evaluated the radiographs to determine a score. Interobserver reliability and Intraobserver reliability was statistically calculated using the interclass correlation co-efficient (ICC).

Results: This was a longitudinal cohort study involving 60 patients who were treated for open tibial fractures. At 6 months the median RUST scores for all 60 patients were 9.5. At 6 months the interobserver agreement was excellent and statistically significant (ICC 0.911).

For the prospective cases, The Inter-rater agreement amongst observers for RUST score was found to be good and statistically significant at 6 weeks (ICC 0.872) that improved to excellent at 6 months (ICC 0.962).

Discussion: Radiological assessment of fracture healing is accepted universally due to its availability and low costs. However, it is still a subjective interpretation of data and can be misleading. The RUST score tends to address these shortcomings by applying a numerical value to various parameters visible on radiographs. To avoid the pitfall of subjectivity, the score has been subsequently validated in studies applying inter and intra observer reliability when independently observed by number of evaluators. This study tends to confirm that there is an almost perfect agreement amongst observers across various specialties when the RUST score is applied for open tibial fractures treated with intramedullary nailing.

Keywords- Tibial fractures, RUST score, Intramedullary nailing, Interobserver reliability.
\end{abstract}




\section{Introduction}

Tibia is the commonest long bone to be fractured with a frequency of about 26 fractures per 100,000 population per year. Tibial fractures are approximately three times more common in males than in females. The average age of patients who sustain a tibial fracture is thirty-seven years. In males, tibial fracture are more common in young adults and are generally attributed to higher energy trauma such as motor vehicular accidents. The subcutaneous position of the tibia leads to higher incidence of fractures and to less soft tissue coverage, there is an increased incidence of impaired healing. Impaired fracture healing leads to prolonged disability, with extra burden on the patient $^{(1)}$.

Progression of healing in diaphyseal tibial fractures is determined by clinico-radiological findings but there is no universally accepted method to evaluate fracture union. Recently researchers at the University of Toronto and McMaster University have developed a radiographic union score for tibia (RUST). The score is evaluated on two orthogonal radiographs for appearance of bridging callus and disappearance of fracture line ${ }^{(2-8)}$.

The aim of this study was to evaluate the interobserver and intra-observer reliability of RUST score amongst four observers ( 2 radiologists and 2 orthopedic surgeons) in open tibial fractures treated with intramedullary nail. Most studies in literature have used a variable mixture of closed and open tibial fractures to apply the RUST score. The treatment modalities used have also been diverse. We have used the RUST score for only open tibial fractures treated with a single modality of stabilization.

\section{Materials and Methods}

This is a longitudinal study approved by the institutional ethics committee. Prior to enrolment in the study, informed consent was obtained from all patients after explaining the whole procedure to them. We included 60 patients with open fracture of tibial diaphysis treated with intramedullary nail with two sets of anteroposterior (AP) and lateral digital radiographs of the leg including the knee and ankle joins done on Discovery XR-650/656 digital X-ray machine by GE with bucky at $100 \mathrm{~cm}$ from the limb and printed on a $14 \times 17$ " film.

Of the 60 patients, forty were prospective and 20 retrospective over a period of 2 years. For retrospective cases $\mathrm{X}$-rays were done at 6 months post-operative period, as this was considered ideal to define fracture healing.

Patients with pathological fractures, closed fractures and open tibial fractures treated with a method of stabilization different from intramedullary nail, patients with chronic arthritis, vascular disorders or peripheral neuropathy and patients with another fracture in the same extremity were excluded from the study.

The RUST scoring system was applied based on the assessment of fracture healing at each of the four cortices (i.e. medial and lateral cortices on the antero-posterior X-ray, anterior and posterior cortices on the lateral X-ray), each cortex received a score of:

1 point-if there was a fracture line with no callus

2 points- if there was a fracture line but with visible callus and

3 points- if there was a bridging callus and fracture line not invisible.

The individual cortical scores were added to give a total score of 4 , which was the minimum score indicating that the fracture union was incomplete and 12 being the maximum indicating that the fracture had healed completely. Radiographic union was defined when the callus was evident on at least 3 cortices on standard AP and lateral radiographs with RUST score of $\geq 7$

It was a blind study with 2 radiologists and 2 orthopedic surgeons who independently viewed the radiographs. They were not involved in the selection of radiographs and were blinded to patient's history; age of the fracture and to each others interpretation of the films.

Inter-observer reliability was evaluated by comparing the scores given by the observers 
separately at the initial viewing of the radiographs at 6 weeks and at 6 months after surgery. Intraobserver reliability was achieved by taking the RUST scoring of the radiographs again at an average period of 6 weeks (range 2-8 weeks) after the initial assessment.

Statistical Analysis- the data collected was entered in Microsoft Excel and statistical analysis was performed using SPSS software for Windows program (21.0 version). The continuous variables were evaluated with mean $( \pm \mathrm{SD})$ or range value when required for comparison of the means between the observers, interclass correlation coefficient (ICC) with its $95 \%$ confidence was used. A $\mathrm{p}$ value of less than 0.001 was regarded as significant. ICC was interpreted as follows: -

0-0.2 indicates poor agreement

0.3-0.4 indicates fair agreement

$0.5-0.6$ indicates moderate agreement

0.7-0.8 indicates strong agreement

$>0.8$ indicates almost perfect agreement

\section{Observations and Results}

This was a longitudinal cohort study involving 60 patients of open tibial fractures treated with an interlocking nail. 20 retrospective and 40 prospective cases were included in the study. Out of these $52(87 \%)$ were male patients and the rest were females. Mean age of the patients was $36+_{-}$ 13.69 (range 18-80 years). The highest number of patients $(36.67 \%)$ belonged to the age group 2130 years.

At six months the RUST score for all 60 patients ranged from 4-12 with a mean score of $9.433+_{-}$ 1.98 (median score 9.5) with maximum number of radiographs (14) showing a score of 9 (Fig 1).

Inter-observer agreement at six weeks for the first observation and for prospective cases was found to be good and statistically significant with an Interclass correlation (ICC) of $0.872 \quad(95 \%$ confidence interval: 0.781-0.928). For the second observation at six weeks, Inter-observer agreement was also good with an ICC of 0.820 (95\% confidence interval: 0.676-0.902).
Inter-observer agreement at six months for the first observation and for prospective cases was found to be perfect and statistically significant with an Interclass correlation (ICC) of 0,962 (95\% confidence interval: 0.927-0.980). Inter-observer agreement at six months for the second observation and for prospective cases was found to be perfect and statistically significant with an Interclass correlation (ICC) of $0.956 \quad(95 \%$ confidence interval: 0.928-0.975).

Inter-observer agreement at six months for the first observation and for retrospective cases was found to be perfect and statistically significant with an Intrerclass correlation (ICC) of 0.961 (95\% confidence interval: 0.923-0.983). Interobserver agreement at six months for the second observation and for retrospective cases was found to be perfect and statistically significant with an Interclass correlation (ICC) of $0.911995 \%$ confidence interval: o.826-0.961).

The Inter-rater agreement amongst observers for RUST score was found to be good and statistically significant at 6 weeks (ICC 0.872) that improved to excellent at 6 months (ICC 0.962). The interobserver agreement was excellent and statistically significant at 6 months for retrospective cases (ICC 0.961).

The Intra-observer reliability of the RUST score was found to be good and statistically significant at 6 weeks (ICC 0.820 ) that improved to excellent at 6 months (ICC 0.956). At 6 months, the intraobserver reliability of RUST score was excellent and statistically significant (ICC 0.911) (Fig 2-8). 


\section{JMSCR Vol||05||Issue |10||Page 28544-28552||October}
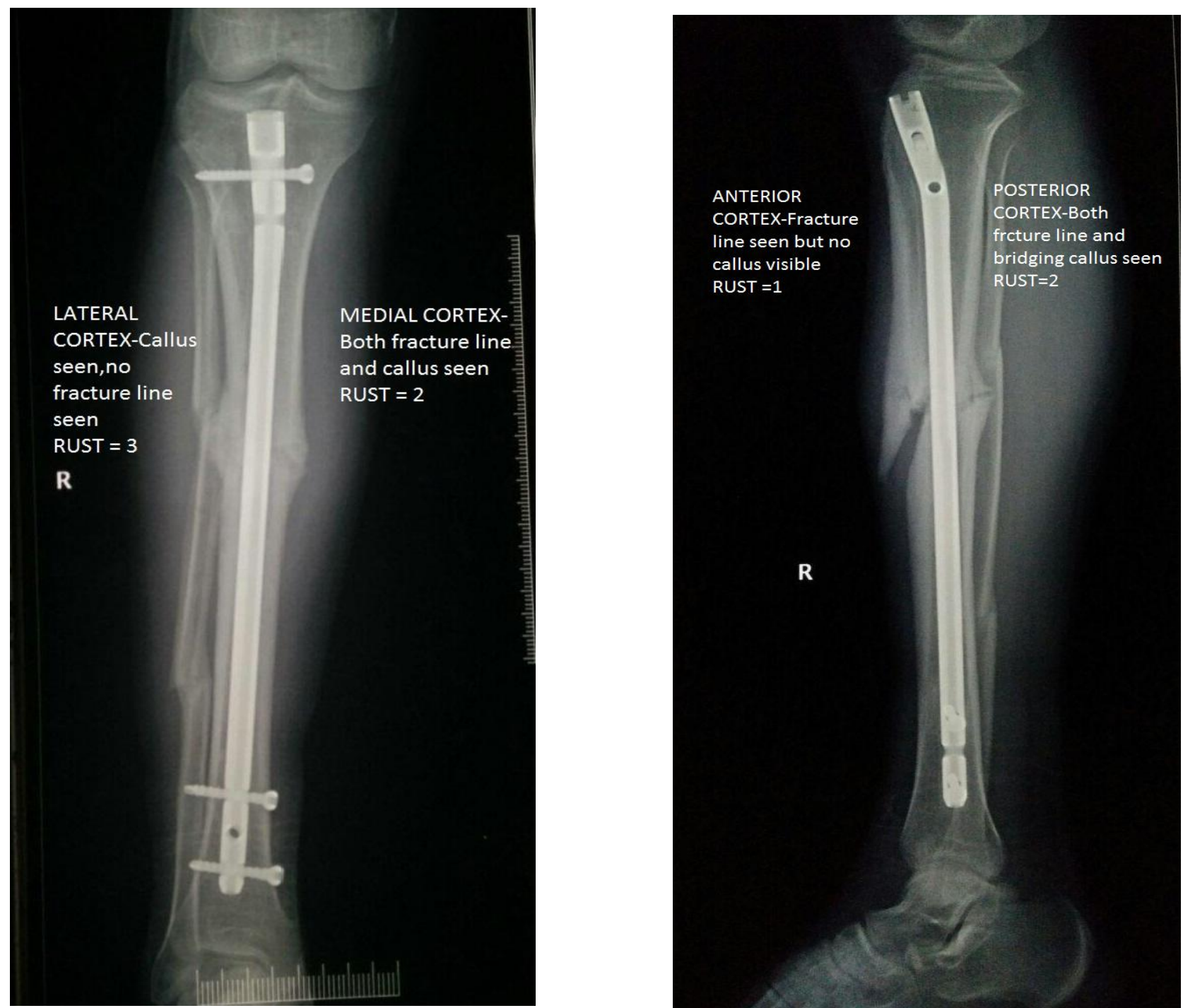

Fig 1. Anteroposterior and lateral radiographs showing the Application of the RUST score for a tibial fracture treated with intramedullary nail

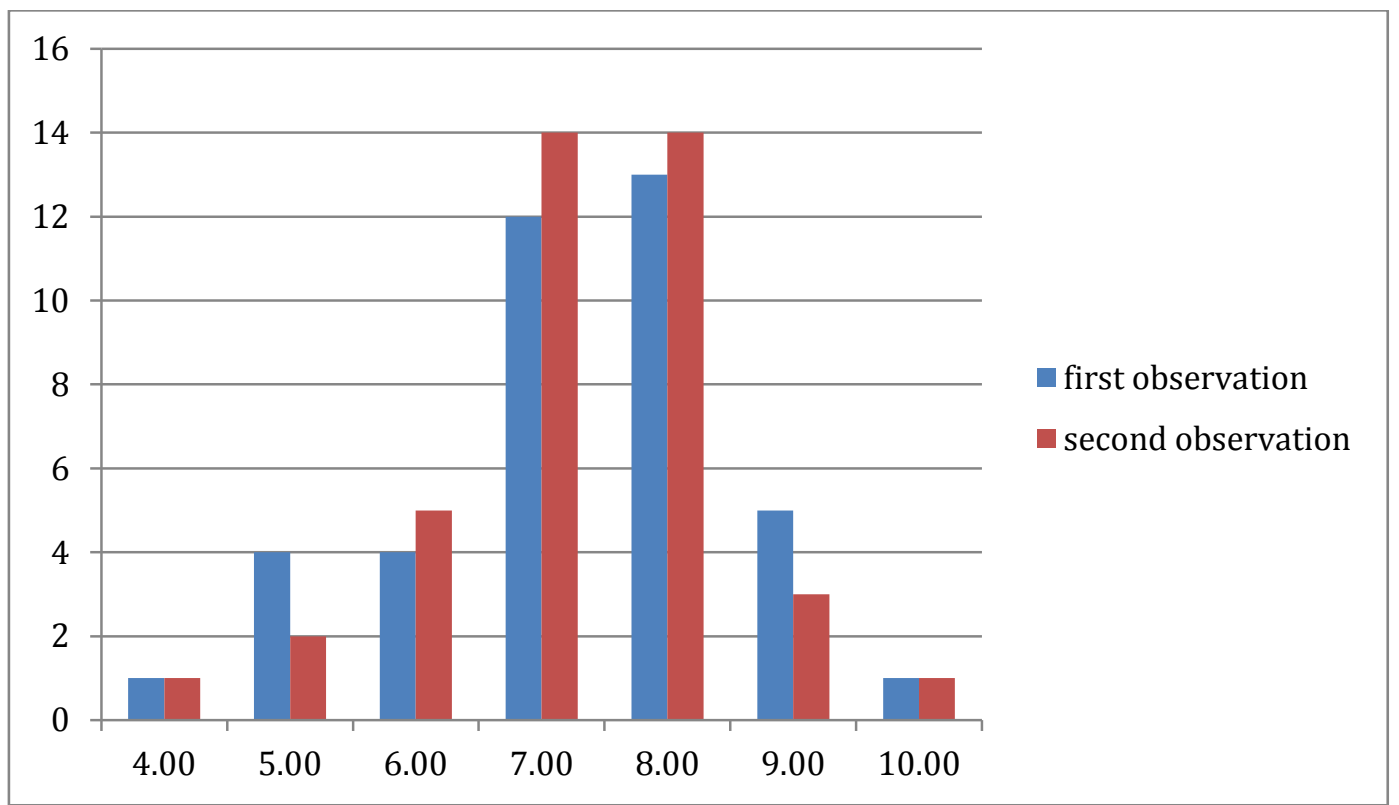

Fig 2 Intraobserver scores by the first orthopedic surgeon for prospective cases at 6 weeks 


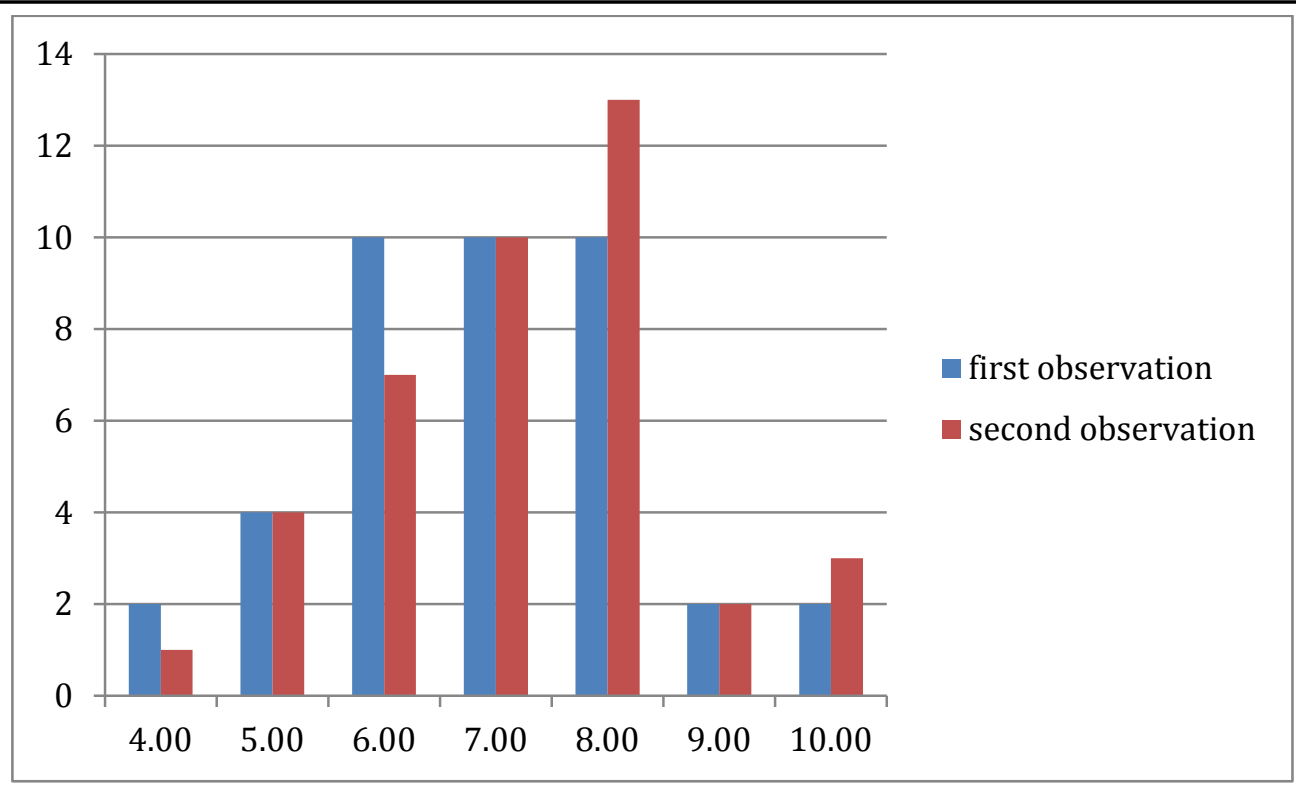

Fig 3. Intraobserver scores by the second orthopedic surgeon for prospective cases at 6 weeks

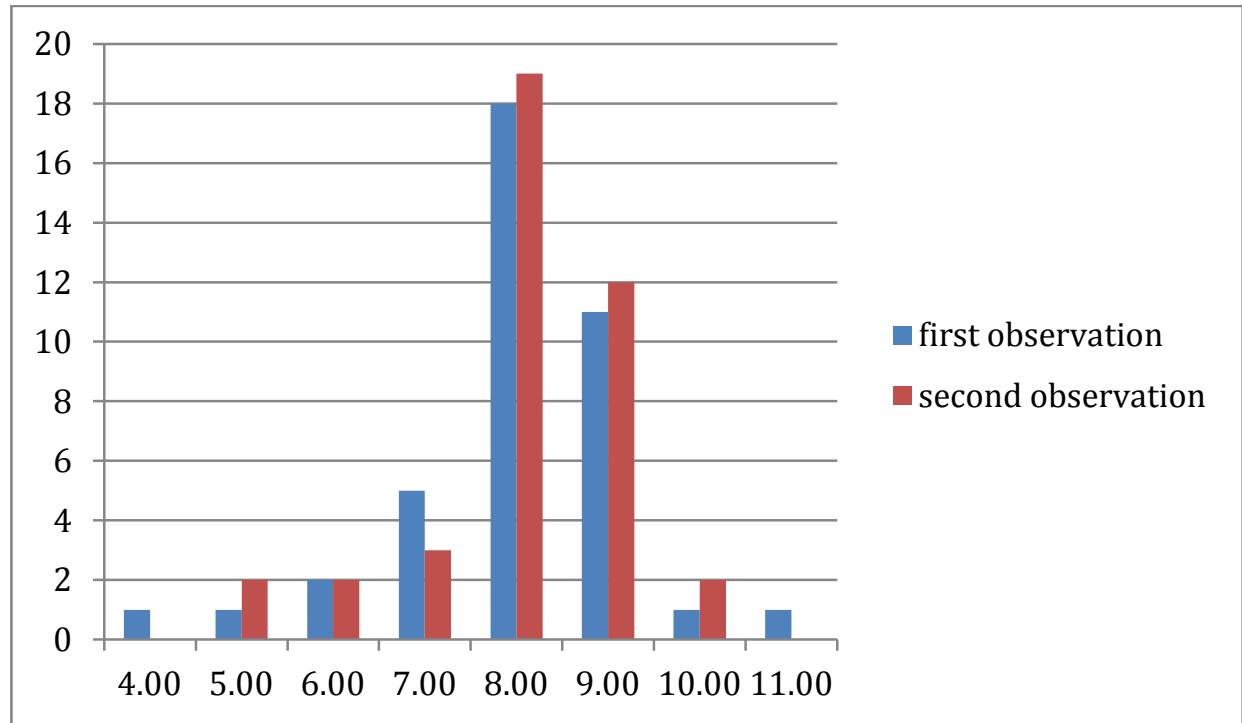

Fig 4 Intraobserver scores by the first radiologist for prospective cases at 6 weeks

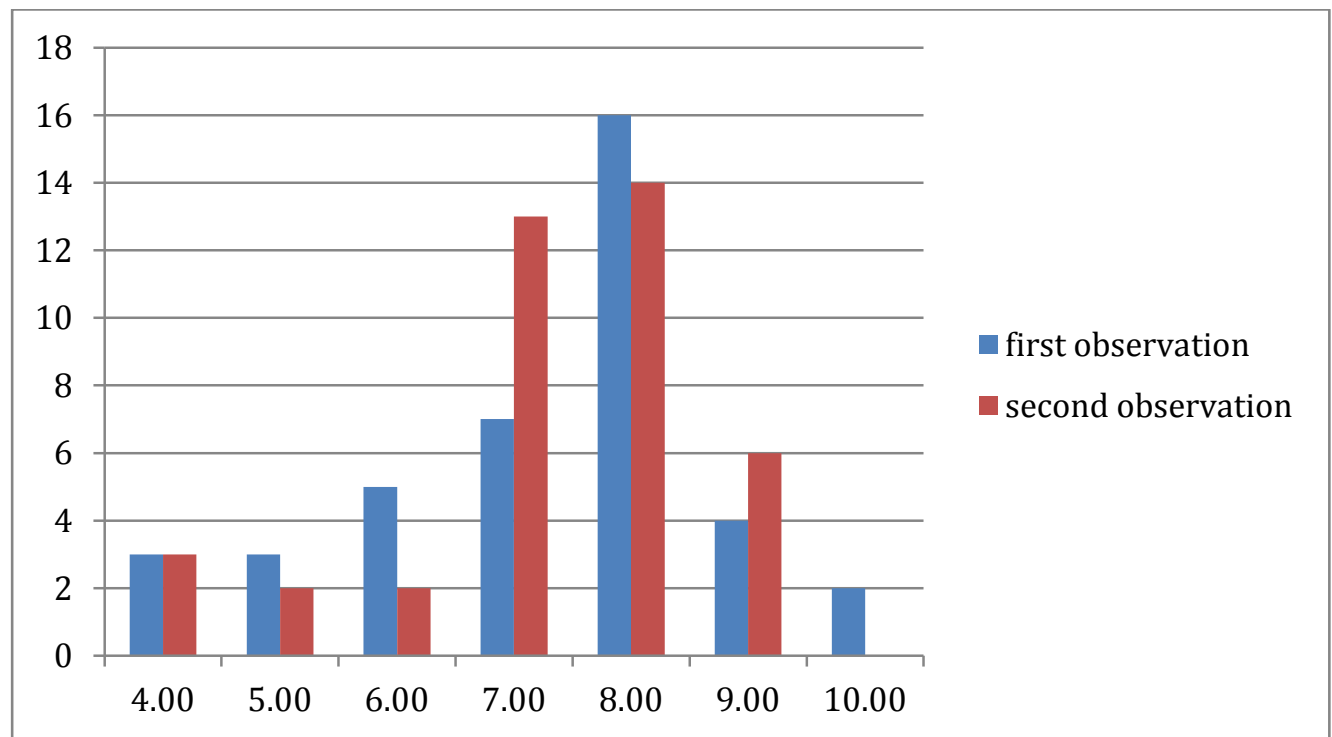

Fig 5 Intraobserver scores by the second radiologist for prospective cases at 6 weeks 


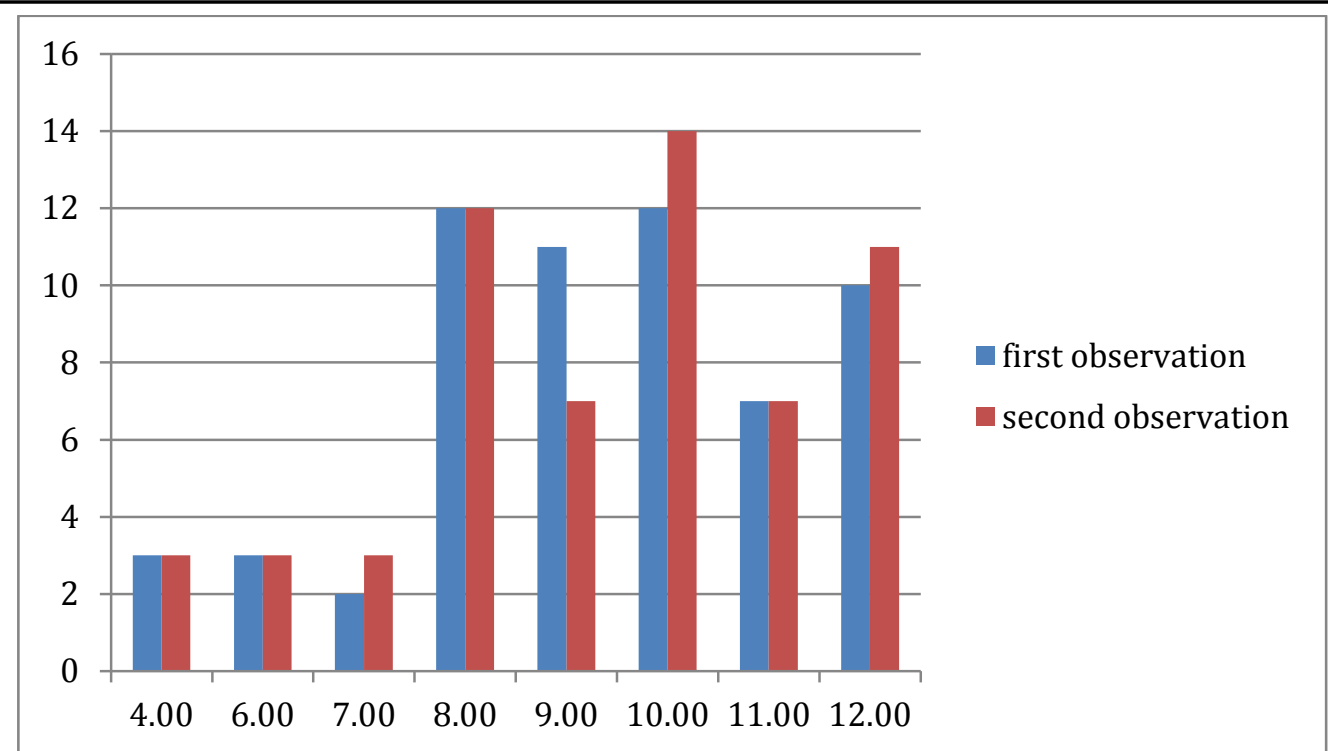

Fig 6 Intraobserver scores by the first orthopedic surgeon for all cases at 6 months

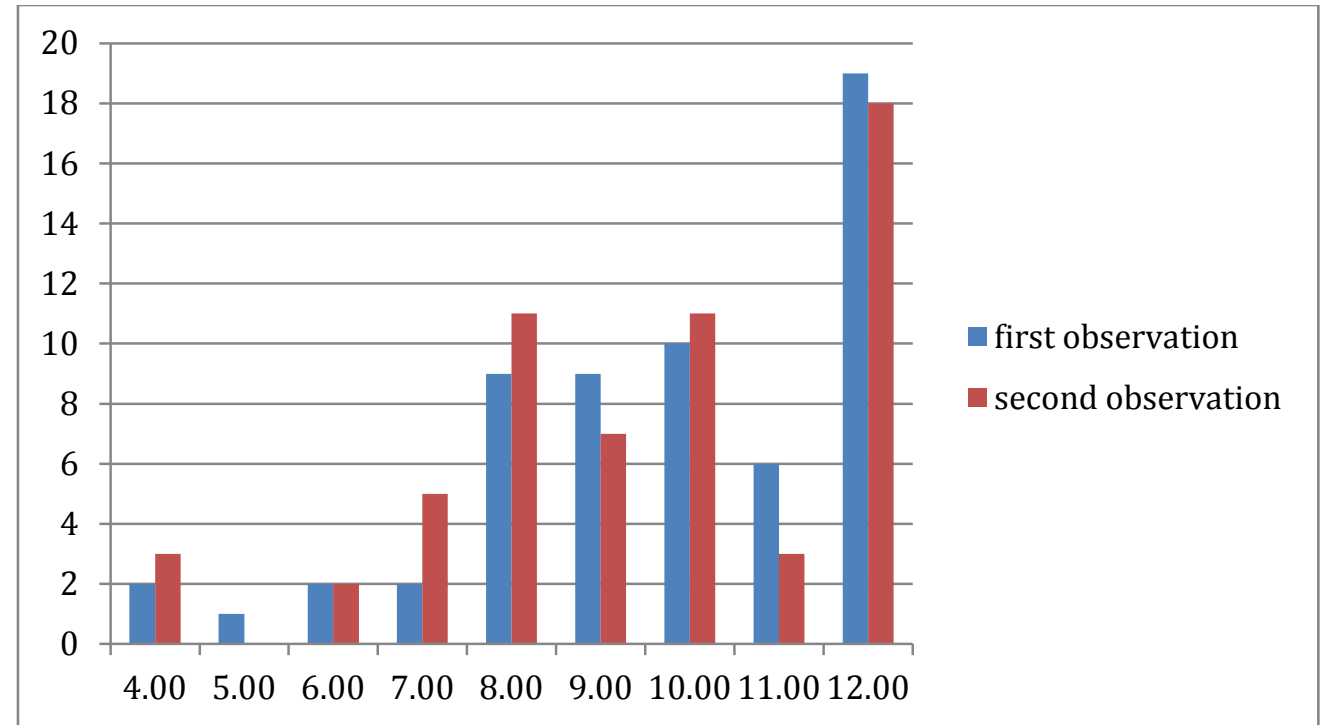

Fig 7 Intraobserver scores by the second orthopedic surgeon for all cases at 6 months

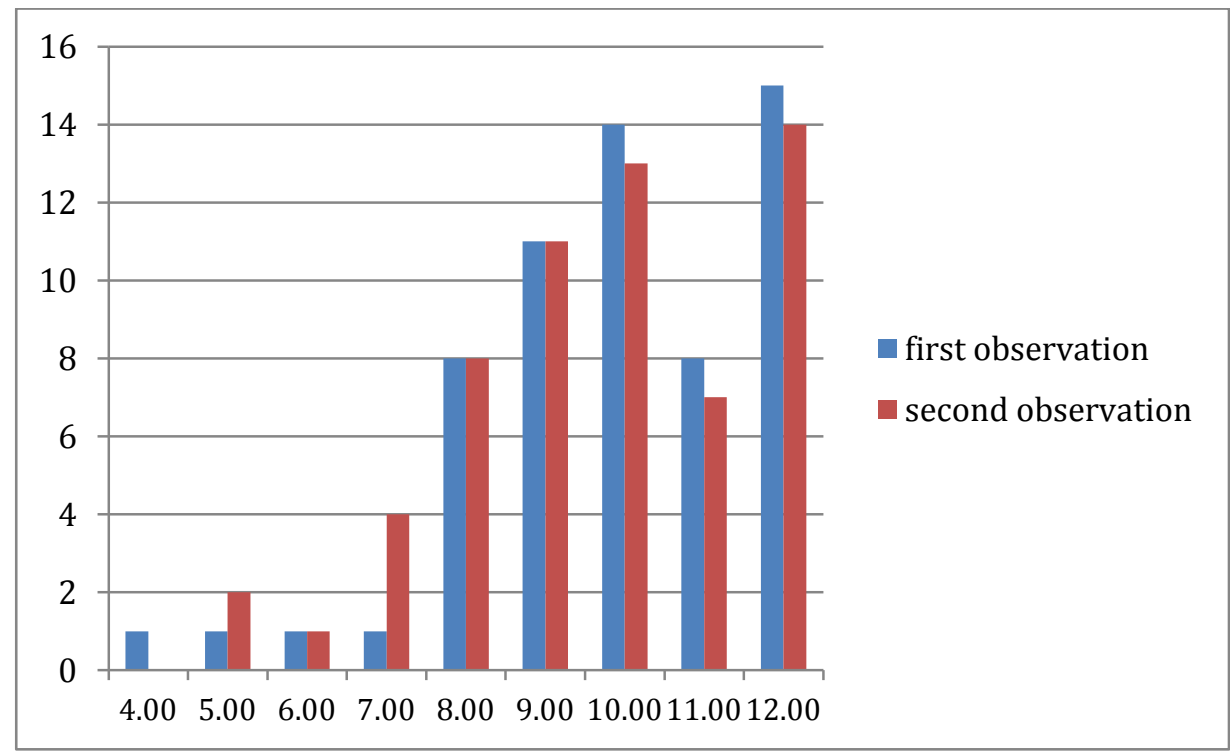

Fig 8 Intraobserver scores by the first radiologist for all cases at 6 months 


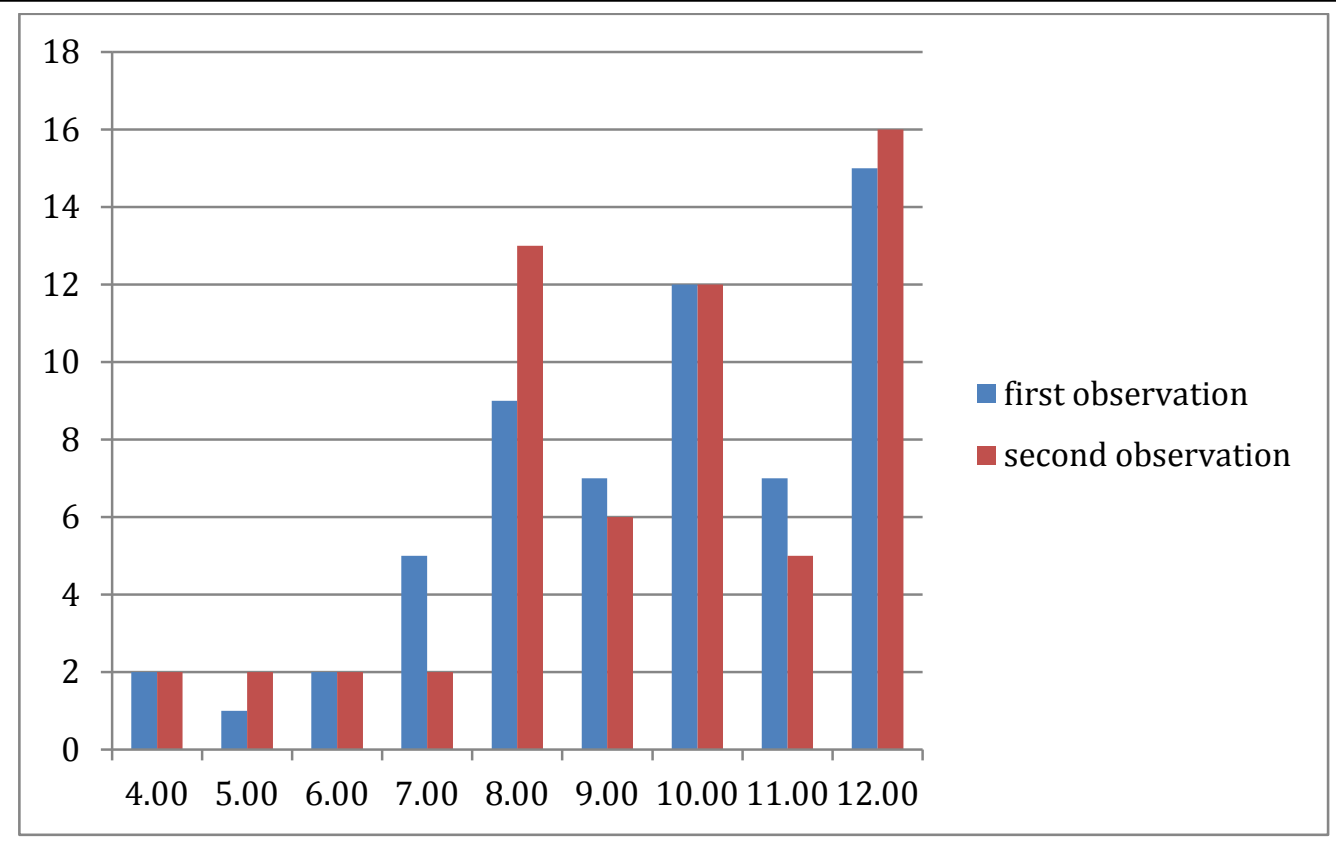

Fig 9: Intraobserver scores by the first radiologist for all cases at 6 months

\section{Discussion}

In spite of numerous subjective and objective methods available in literature to assess fracture healing and consolidation, a reliable and gold standard method is yet to be established ${ }^{(2-8)}$. In literature we find a combination of clinical and radiological parameters used to assess the progress of fracture union. Various authors have used these parameters subjectively to define fracture healing but what has been lacking is an objective method to quantify the same. Modalities such as ultrasonography, radionuclide imaging and resonant frequency analysis have been used primarily in research settings and are either too cumbersome or too expensive to be used routinely in clinical practice ${ }^{(9,10,11)}$.

Wheelan et al evaluated the inter-observer and intra- observer reliability for the radiographic union score by Hammer et al ${ }^{(4,6)}$. They used the following criteria: "General impression" of healing, number of cortices bridged by callus and number of cortices showing a fracture line. There was a moderate agreement amongst observers for surgeon's " General impression" $(\kappa=0.65,95 \% \mathrm{CI}$ $0.59-0.75)$ and Hammer scale $(\kappa=0.6,95 \% \mathrm{CI}$ $0.52-0.68)$. The inter- observer reliability was highest for number of cortices showing bridging callus $((\kappa=0.75,95 \%$ CI $0.61-0.89)$ and number of cortices showing fracture line $((\kappa=0.70,95 \% \mathrm{CI}$ $0.56-0.84)$ but the overall agreement was moderate. These observations demanded for the development of a better, simpler and more reliable radiological scoring method. Subsequently, the same authors described the RUST score ${ }^{(8)}$. They evaluated 45 sets of radiographs of closed tibial shaft fractures treated with intramedullary fixation. Seven orthopedic reviewers independently evaluated the radiographs. Overall agreement was substantial (ICC 0.86, 95\% CI 0.79$0.91)$. The reliability improved when the observations were made by traumatologists compared to general orthopedic surgeons (ICC 0.86, 0.81 and0.83 respectively). Kooistra et al confirmed the strong inter- observer reliability of RUST score in aprospective study on 549 sets of tibial radiographs (ICC 0.84, 95\% CI 0.80-0.87) (12). Leow reported a strong agreement between five evaluaters with $\mathrm{ICC}=0.75$ (95\% CI 0.65 0.84). They also found that the ICC increased from 0.75 to 0.79 when the postoperative radiographs were available.

Azevedo et al reported a ICC of 0.87 (95\% CI 0.81-0.91) among evaluators. The reliability increased as the experience of the evaluator improved: greater reliability amongst traumatologists compared to first, second and third year 
residents (ICC 0.94, $0.80,0.92$ and 0.90 respectively) ${ }^{(5,16,17)}$.

The behavior of Implant bone complex leading to fracture healing has been studied in experimental conditions $^{(18)}$. This simulation showed a secondary pattern of bone healing. In the initial phases, bone formation occurred away from the fracture gap in the form of external callus. Subsequently the bone formation occurred in the fracture gap itself.

In the initial phases of healing, the radiographic interpretation should yield a RUST score of no more than 8 as disappearance of fracture line cannot happen in these phases.

Radiographic interpretation of fracture healing based on presence of external callus and persistence or disappearance of fracture line is also subject to fallacy. In oblique and spiral fractures or oblique tibial osteotomies, the overlap of fracture gap and normal bone in AP and Lateral radiographs has resulted in false interpretation of healing status ${ }^{(19)}$.

In open tibial fractures, one or more cortices may be lost. This is likely to result in a lower RUST score with the maximal score not exceeding 9 even for a fully consolidated fracture.

Radiological union score for tibia is a validated instrument to determine radiological union in tibial fractures. This study confirmed that RUST score features perfect agreement on inter-observer and intra-observer reliability and compliance. The inter-observer and intra-observer reliability improved with successive observations.

\section{References}

1. Bhandari M, Guyatt G, Swiontkowski M, schemitsch E. treatment of open fractures of the shft of the tibia. J Bone Joint Surg 2001;83(1): 62-68

2. Panjabi M, Walter S, Karuda M, white A, Lawson J. Correlations of radiographic analysis of healing fractures with strength: A statistical analysis of experimental osteotomies. J Orthop Res 1985; 3 (2): 212-218
3. Corrales L, Morshed S, Bhandari M, Miclau T. variability in the assessment of fracture healing in orthopedic trauma studies. J Bone joint Surg 2008; 90 (9): 1862-1868

4. Hammer R, Hammerby S, Lindholm B. Accuracy of radiologic assessment of tibial shaft fracture union in humans. Clin Orthop Relat Res 1985; 199: 233-238

5. Lane J, Sandhu H. Current approaches to experimental bone grafting. Orthop Clin North Am 1987; 18: 213-225

6. Whelan D, Bhandari M, Mckee M, Guyatt G, Kreder H, Stephan D et al. Interobserver and Intra-observer variation in the assessment of healing of tibial; fractures after intramedullary fixation. J Bone Joint Surg 2002; 84 (1): 15-18

7. Bhandari M, Guyatt G, Swiontkowski M, Tornetta P, Sprague S, Schemitsch E. A lack of consensus in the assessment of fracture healing amongst orthopedic surgeons. J Orthop Trauma 2002; 16 (8): 562-566

8. Whelan D, Bhandari M, Stephan D, Kreder H, Mckee M, Zdero $\mathrm{R}$ et al. Development of the radiographic union Score for tibial fractures for the assessment of tibial fracture healing after intramedullary fixation. J Trauma Injury Infect Crit Care 2010; 68 (3): 629-632

9. Krestan CR, Noske H, Vasilevska V, Weber M, Scheuller G, Imhof H, Czerny C.MDCT Versus Digital radiography in the evaluation of bone healing in Orthopedic patients. Am J Radiol 2006; 186:1754-1760

10. Smith MA, Jones EA, Strachan RK, Nicoll JJ, Tothill P, Hughes SPF. Prediction of fracyure healing in the tibia by quantitative radionuclide imaging. $\mathrm{J}$ Bone Joint Surg1987; 69 (3): 441-447

11. Claes LE, Cunningham JL. Monitoring the mechanical properties of healing bone. 
Clin Orthop Relat Res 2009; 467 (8): 1964-1971

12. Cekic E, Alici E, Yesil M. Reliability of radiographic union score for tibial fractures. Acta Orthop Traumatol Turc 2014; 46 (4): 746-750

13. Kooistra BW, Dijkamn BG, Busse JW, Sprague S, Schemitsch EH, Bhandari M. The radiographic union score in tibial fractures: Relaibility and Validity. J Orthop Trauma 2010; 24 Suppl. 3: S81-86

14. Leow J, Clement N, Tawonsawatruk T, Simpson C, Simpson A. The radiographic union scale in tibial (RUST) fractures: Reliability of the outcome measure at an imndependant center. Bone Joint Res 2016; 5(4): 116-121

15. Azevedo FF, Cotias R, Azi M, Teixiera A. Reliability of the radiographic union scale in tibial fractures (RUST). Revista Brasileira de Orthopedia 2017; 52 (1): 35-39

16. Mohit B, Bauke W, Jason B, Stephan W, Paul T, Emil S. Radiographic union scale for tibial (RUST) fracture healing assessment: preliminary validation. J Bone Joint Surg 2011; 93 Suppl. IV: 575

17. Tawonsawatruk T, Hamilton DF, Simpson HRW. Validation of the use of Radiographic fracture-healing score in a small animal model. J Orthop Res 2014: 1117-1119

18. Steiner M, Claes L, Ignatius A, Simon U Wehner T. Optimization of intramedullary nailing by numerical simulation of fracture healing. J Orthop Res 2012; 30(4): 569573

19. Chehade MJ, Vakaci IA, Callary SA, Findlay DM, Solomon LB. Differentially loaded Radiostereometric Analysis in Torsion adds essential informationin Diaphyseal bone healing: the example of a tibial osteotomy. J Bioengineer \& Biomedical Sci 2011 S1: 004. 